\title{
Catalpol Promotes the Proliferation and Differentiation of Osteoblasts Induced by High Glucose by Inhibiting KDM7A
}

This article was published in the following Dove Press journal: Diabetes, Metabolic Syndrome and Obesity: Targets and Therapy

\author{
Jian Cheng $\mathbb{D}^{1,2, *}$ \\ Hai-yan $\mathrm{Xu}^{3, *}$ \\ Ming-ming $\mathrm{Liu}^{4}$ \\ Jian-ping $\mathrm{Cai}^{2}$ \\ Lei Wang ${ }^{2}$ \\ Zhen $\mathrm{Hua}^{2}$ \\ Xiao-dong $\mathrm{Wu}^{\prime}$ \\ Wei-ling Huo' \\ Nan-ning Lv ${ }^{4}$ \\ 'Department of Orthopedics, Xuzhou \\ Central Hospital Affiliated to Nanjing \\ University of Chinese Medicine, Xuzhou, \\ Jiangsu 221009, People's Republic of \\ China; ${ }^{2}$ Institute of Traumatology \& \\ Orthopedics, Nanjing University of \\ Chinese Medicine, Nanjing, Jiangsu \\ 210029, People's Republic of China; \\ ${ }^{3}$ Department of Human Anatomy, \\ Xuzhou Medical University, Xuzhou, \\ Jiangsu 221004, People's Republic of \\ China; ${ }^{4}$ Department of Orthopedic \\ Surgery, Lianyungang Second People's \\ Hospital, Lianyungang 222023, People's \\ Republic of China \\ *These authors contributed equally to \\ this work
}

Correspondence: Ming-ming Liu Department of Orthopedic Surgery, Lianyungang Second People's Hospital, No. 4I Hailian East Road, Haizhou District, Lianyungang, Jiangsu 222023, People's Republic of China Email mmliu89@I26.com

Jian-ping Cai

Institute of Traumatology \& Orthopedics, Nanjing University of Chinese Medicine, Nanjing, Jiangsu 210029, People's Republic of China

Email caijianpingcjp@I26.com
Introduction: The protective effect of catalpol on diabetic osteoporosis (DOP) and its mechanism remain unclear. This study aimed to explore whether catalpol enhanced the proliferation and differentiation of MC3T3 cells induced by high glucose by inhibiting the expression of KDM7A.

Methods: MC3T3 cells were induced by high glucose (HG) and treated with different concentrations of catalpol. The proliferation and mineralization abilities of MC3T3-E1 cells were determined by CCK- 8 assay and Alizarin Red Staining, respectively. The expression of differentiation-related osteogenic proteins, KDM7A and related proteins of $\mathrm{Wnt} / \beta$-catenin signaling pathway was analyzed by Western blot analysis. The alkaline phosphatase (ALP) activity was detected by ALP assay kits.

Results: MC3T3-E1 cells induced by high glucose showed decreased proliferation and mineralization abilities and decreased ALP activity, which were all reversed by the treatment of catalpol. High glucose induction inhibited the expression of KDM7A, Total- $\beta$-catenin, Nuclear- $\beta$-catenin and p-GSK3 $\beta$, which was reversed by the treatment of catalpol. And KDM7A interference up-regulated the expression of Total- $\beta$-catenin, Nuclear- $\beta$-catenin and p-GSK3 $\beta$, which was down-regulated by KDM7A overexpression. Furthermore, the proliferation and mineralization abilities and ALP activity were improved when treated with KDM7A interference and decreased when treated with KDM7A overexpression. However, SKL2001 could improve the proliferation and mineralization abilities and ALP activity of MC3T3-E1 cells.

Discussion: Catalpol promotes the proliferation and differentiation of osteoblasts induced by high glucose by regulating the Wnt/ $\beta$-catenin signaling pathway through KDM7A.

Keywords: catalpol, KDM7A, proliferation, differentiation, osteoblasts, high glucose

\section{Introduction}

Diabetes mellitus (DM) is a chronic metabolic syndrome characterized by hyperglycemia. In the world, high incidence of DM seriously threatens public health in the 21 st century. ${ }^{1}$ Diabetes is a disease of the endocrine system, which can cause complications of the eyes, nerves, feet, kidneys, nervous system, heart and skeleton, which has brought a great blow to DM patients and their families. ${ }^{2}$ With osteoporosis as the main manifestation of DM, diabetic osteoporosis (DOP) is a metabolic bone disease with high incidence belonging to DM complications. Diabetic patients are affected by factors such as abnormal insulin and obesity. The lack of insulin will cause the imbalance of bone resorption and bone formation, 
which will further aggravate osteoporosis. ${ }^{3,4}$ Due to unclear pathogenesis of DOP, the treatment effect of it is not ideal. Therefore, it is urgent to find an effective method to alleviate the osteoporosis of DM patients.

Catalpol is a kind of active ingredient mainly composed of iridoid, and it is extracted from the roots of Rehmannia glutinosa. Traditional Chinese medicine (TCM) has long been used to treat DM, osteoporosis, and cardiovascular disease in China. ${ }^{5}$ Catalpol has been widely studied in DM complications. Zou et $\mathrm{al}^{6}$ indicated that catalpol could mitigate myocardial damage and exert cardioprotective effect against diabetic cardiomyopathy. Studies have shown that catalpol could improve neointimal hyperplasia in hyperglycemic rats. ${ }^{7,8}$ And, catalpol could reduce the injury of diabetic atherosclerosis. ${ }^{9}$ Furthermore, the bone healing capacity of bone marrow mesenchymal stem cells (BMSCs) was improved by the catalpol treatment through promoting the BMSC proliferation and osteogenic differentiation. ${ }^{10,11}$ However, the role of catalpol in diabetes-induced osteoporosis has not been studied. Recent studies have shown that KDM7A interference can promote BMSCs to differentiate into osteoblasts by activating the $\mathrm{Wnt} / \beta$-catenin signaling pathway. ${ }^{12}$

In this study, we constructed the model of osteoblast MC3T3-E1 cells induced by high glucose. This study was designed to explore whether catalpol could regulate the proliferation and differentiation of MC3T3 cells induced by high glucose through activating the $\mathrm{Wnt} / \beta$-catenin signaling pathway through KDM7A.

\section{Materials and Methods}

\section{Cell Culture and Cell Induction}

MC3T3-E1 cells were purchased from American Type Culture Collection (ATCC ${ }^{\circledR}$ CRL-2594; Rockville, MD, USA). MC3T3-E1 cells were placed in $\alpha$-MEM medium containing $10 \%$ fetal bovine serum (FBS), $100 \mathrm{IU} / \mathrm{mL}$ penicillin and $100 \mu \mathrm{g} / \mathrm{mL}$ streptomycin and cultured at $37^{\circ} \mathrm{C}$ with $5 \% \mathrm{CO}_{2}$ under saturated humidity. The medium was replaced every 2-3 days, and MC3T3-E1 cells had digestive transfer culture with $0.25 \%$ trypsin after fusion growth. MC3T3-E1 cells were induced by high glucose (HG) for $14 \mathrm{~d}$ and treated with different concentrations of catalpol (Purity 98\%, Chengdu Dast Biotechnology Co., Ltd) $(1 \mathrm{mg} / \mathrm{mL}, 2 \mathrm{mg} / \mathrm{mL}$ and $4 \mathrm{mg} / \mathrm{mL})$. MC3T3-E1 cells were treated with mannitol in HM group while received no treatment in the control group. Mannitol was used as a control group of osmotic pressure.

\section{Cell Transfection}

After induction of high glucose for $14 \mathrm{~d}$, MC3T3-E1 cells were, respectively, transfected with shRNA-NC, shRNAKDM7A-1, shRNA-KDM7A-2, overexpression-NC and overexpression-KDM7A using Lipofectamine ${ }^{\circledR} 2000$ reagent (Invitrogen; Thermo Fisher Scientific, Inc., Waltham, MA, USA). The transfection efficiency was determined by Western blot analysis. Moreover, HG-induced MC3T3-E1 cells transfected with overexpression-KDM7A were also treated with SKL2001 (Wnt/ß-catenin agonist).

\section{CCK-8 Assay}

MC3T3-E1 cells were inoculated into a 96-well plate (100 micron) at the density of $5 \times 10^{3}$ cells per well. The holes around the inoculated cells were added with sterile PBS, and they were considered as the blank control. After the high glucose induction and catalpa treatment, $10 \mu \mathrm{L}$ CCK-8 reagent was added to each hole and incubated in the incubator for $3 \mathrm{~h}$ under dark conditions. The 96-well plate was put into the enzyme-linked immunometric meter, and the absorbance (OD value) of each well was measured at the wavelength of $450 \mathrm{~nm}$.

\section{Alizarin Red Staining}

MC3T3-E1 cells were induced with high glucose for $14 \mathrm{~d}$ and treated with catalpa $(1 \mathrm{mg} / \mathrm{mL}, 2 \mathrm{mg} / \mathrm{mL}$ and $4 \mathrm{mg} / \mathrm{mL}$ ). After the culture, MC3T3-E1 cells were washed with precooled PBS, fixed with $70 \%$ alcohol for $20 \mathrm{~min}$, and the fixative solution was discarded. The cell samples were washed with PBS twice, and $500 \mu \mathrm{L} 0.1 \%$ alizarin red dye was added to cover the MC3T3-E1 cells for staining for 5 min. Finally, the staining solution was absorbed and washed with PBS twice. The formation of stained calcified nodules was observed under an inverted phase-contrast microscope.

\section{Western Blot Analysis}

After the induction of high glucose and treatment of catalpa, proteins in MC3T3-E1 cells were extracted with RIPA lysis buffer (Beyotime, China) and protein concentration was determined by Bradford method. Each hole was loaded with $30 \mu \mathrm{g}$ protein, separated with the SDS-PAGE gel for electrophoresis and transferred to nitrocellulose membranes. The 5\% skim milk solution was prepared with the solvent of PBST, which was used to seal the nitrocellulose membranes at room temperature for $2 \mathrm{~h}$. Then, primary antibodies including RUNX2 (ab192256;Abcam), Collagen 
I (ab34710;Abcam), OCN (ab13420;Abcam), BMP4 (ab39973;Abcam), BMP7 (ab56023;Abcam), KDM7A (A14692;ABclonal), $\beta$-catenin (ab16051;Abcam), pGSK3 $\beta$ (ab75745;Abcam), GSK3 $\beta$ (ab32391;Abcam), histone (ab1220;Abcam) and GAPDH (ab9485;Abcam) (dilution,1:1000) were incubated with nitrocellulose membranes at $4{ }^{\circ} \mathrm{C}$ for $12 \mathrm{~h}$. PBST was used to wash the nitrocellulose membranes 3 times with $10 \mathrm{~min} /$ time. HRP-labeled secondary antibody was incubated with the nitrocellulose membranes at room temperature for $1 \mathrm{~h}$. After the nitrocellulose membranes were fully washed, they were then treated with ECL chemiluminescent solution from Millipore. The BioRad gel imaging analysis system was used to collect and analyze the bands.

\section{Alkaline Phosphatase (ALP) Activity}

MC3T3-E1 cells were induced by high glucose for $14 \mathrm{~d}$ and treated with catalpa. After $72 \mathrm{~h}$, PBS solution was added to each hole to wash the plates for 3 times. $0.2 \%$ Triton X-100 $(100 \mu \mathrm{L})$ was added to each hole, and the MC3T3-E1 cells were oscillated with an ultrasonic cell disruptor. The cell lysates were collected after centrifugation and stored at $-70^{\circ} \mathrm{C}$. Total protein concentration was measured with the BCA kits. The ALP content was measured with the ALP assay kit, and the relative ALP activity was calculated finally.

\section{Statistical Analysis}

The experimental data presented as mean \pm standard deviation were processed and analyzed by SPSS 22.0 . The statistical method was one-way analysis of variance with Tukey's post hoc test, which was used to compare the differences among multiple groups. The differences have statistical significance when $\mathrm{P}<0.05$.

\section{Results}

\section{Catalpol Alleviates the Inhibition Effects of HG on Proliferation of MC3T3-EI Cells}

The CCK-8 assay was used to detect the cell proliferation of MC3T3-E1 cells disposed by $\mathrm{HG}$ and catalpol (Figure 1). The proliferation of MC3T3-E1 cells treated with mannitol was not statistically changed compared with the control group, while the proliferation of MC3T3-E1 cells was obviously decreased when cells were disposed by HG. With the increasing catalpol dosages, the

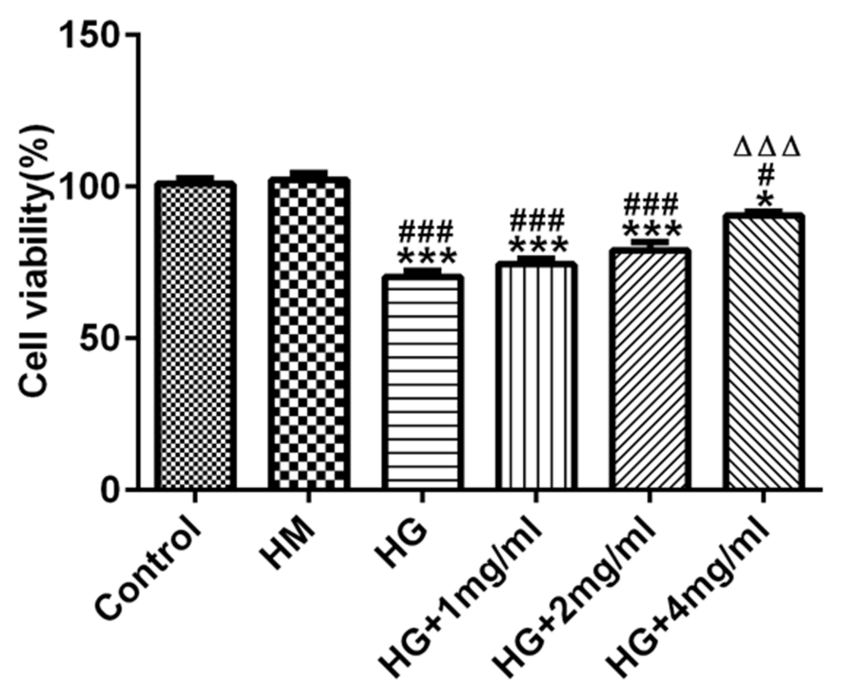

Figure I Catalpol alleviates the inhibition effects of HG on proliferation of MC3T3EI cells. The proliferation of HG-induced MC3T3-EI cells affected by catalpol was determined by $C C K-8$ assay. ${ }^{*} P<0.05$ and ${ }^{* * * P}<<0.001$ vs control group. ${ }^{\#} \mathrm{P}<0.05$ and ${ }^{\#} \mathrm{P}<0.00$ I vs. $\mathrm{HM}$ group. ${ }^{\Delta \triangle \Delta} \mathrm{P}<0.00 \mathrm{I}$ vs. $\mathrm{HG}$ group.

proliferation of HG-induced MC3T3-E1 cells was gradually improved.

\section{Catalpol Alleviates the Inhibition Effects of HG on Differentiation of MC3T3-EI Cells}

As shown in Figure 2A, there is no statistical difference in calcium nodules between control group and $\mathrm{HM}$ group. The calcium nodules were decreased in HGinduced MC3T3-E1 cells while the formation of mineralized nodules was increased when HG-induced MC3T3-E1 cells were treated with the increase of catalpol. The expression of cell differentiation-related proteins was analyzed by Western blot (Figure 2B). The expression of RUNX2, Collagen I, OCN, BMP4 and BMP7 in HG-induced MC3T3-E1 cells was obviously decreased compared with the control group and HM group. However, catalpol treatment promoted the expression of RUNX2, Collagen I, OCN, BMP4 and BMP7 in HG-induced MC3T3-E1 cells and the promotion role of catalpol in the expression of these proteins was enhanced with the increase of catalpol. The ALP activity in the control group was not statistically different from the HM group. HG significantly inhibited the ALP activity in MC3T3-E1 cells and the ALP activity in HG-induced MC3T3-E1 cells was gradually increased when the concentration of catalpol was increased (Figure 2C). 


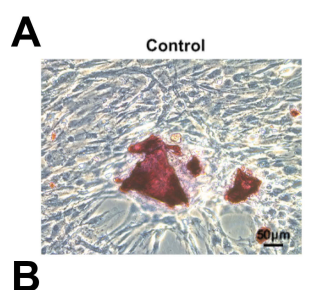

B

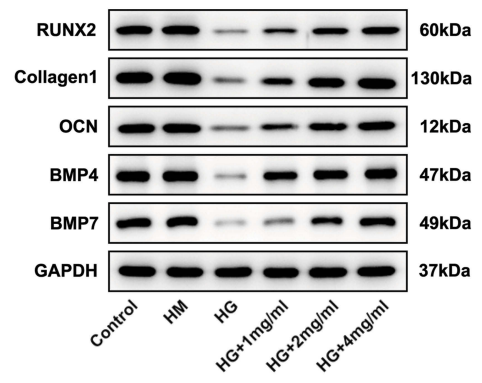

HM

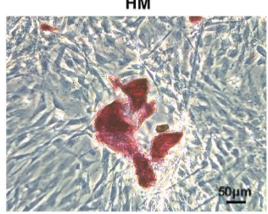

HG

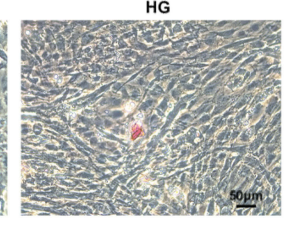

RUNX2

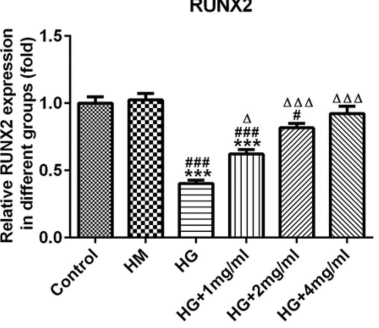

BMP4

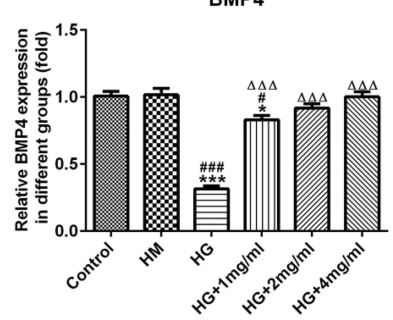

HG+1 $\mathrm{mg} / \mathrm{ml}$

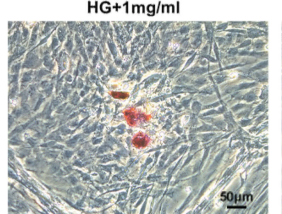

$\mathrm{HG}+2 \mathrm{mg} / \mathrm{ml}$

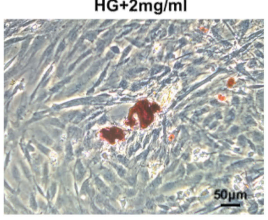

$\mathrm{HG}+4 \mathrm{mg} / \mathrm{ml}$

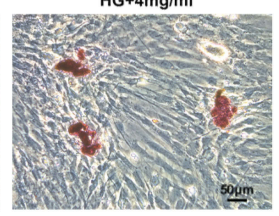

$\mathrm{OCN}$

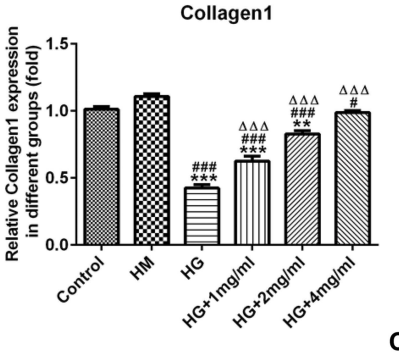

BMP7

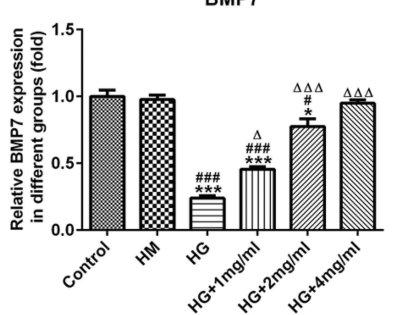

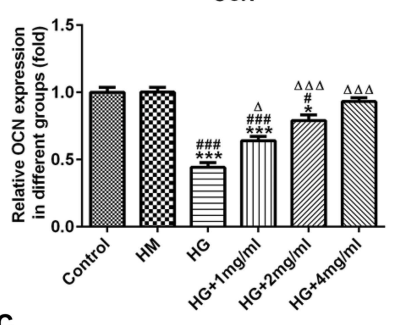

ALP

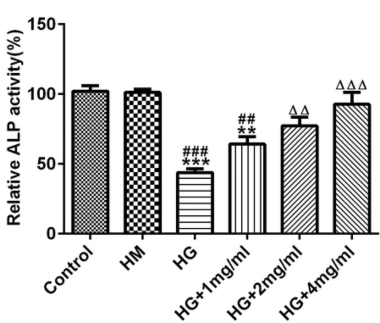

Figure 2 Catalpol alleviates the inhibition effects of HG on differentiation of MC3T3-EI cells. (A) The differentiation of MC3T3-EI cells was observed by alizarin red staining (magnification of 200). (B) The expression of RUNX2, Collagen I, OCN, BMP4 and BMP7 in HG-induced MC3T3-EI cells was detected by Western blot analysis $(\mathrm{n}=3) .{ }^{*} \mathrm{P}<0.05,{ }^{*} * \mathrm{P}<0.0 \mathrm{I}$ and ${ }^{*} * \mathrm{P}<0.00 \mathrm{I}$ vs. control group. ${ }^{\#} \mathrm{P}<0.05$ and ${ }^{\# \#} \mathrm{P}<0.00 \mathrm{I}$ vs. HM group. ${ }^{\Delta} \mathrm{P}<0.05$ and ${ }^{\Delta \Lambda \Delta} \mathrm{P}<0.00 \mathrm{I}$ vs. $\mathrm{HG}$ group. (C) The $\mathrm{ALP}$ activity in $\mathrm{HG}-$ induced MC3T3-EI cells was assessed by the ALP assay kit. ${ }^{* *} \mathrm{P}<0.0 \mathrm{I}$ and ${ }^{* * *} \mathrm{P}<0.00 \mathrm{I}$ vs. control group. ${ }^{\# \#} \mathrm{P}<0.0 \mathrm{I}$ and ${ }^{\# \#} \mathrm{P}<0.00 \mathrm{I}$ vs. $\mathrm{HM}$ group. ${ }^{\Delta \Lambda} \mathrm{P}<0.0 \mathrm{I}$ and ${ }^{\Delta \triangle \Delta} \mathrm{P}<0.00 \mathrm{I}$ vs. HG group.

\section{KDM7A Expression Is Increased by HG Induction, While KDM7A Expression Is Inhibited by Catalpol}

The expression of KDM7A and Wnt/ $\beta$-catenin signaling pathway was analyzed by Western blot. HG upregulated the expression of KDM7A in MC3T3-E1 cells and mannitol could not affect the expression of KDM7A in MC3T3-E1 cells. The increased concentration of catalpol gradually inhibited the expression of KDM7A in HG-induced MC3T3-E1 cells (Figure 3A). The expression of Total- $\beta$-catenin, Nuclear- $\beta$-catenin and p-GSK3 $\beta$ was decreased in HG-induced MC3T3-E1 cells while
A
B

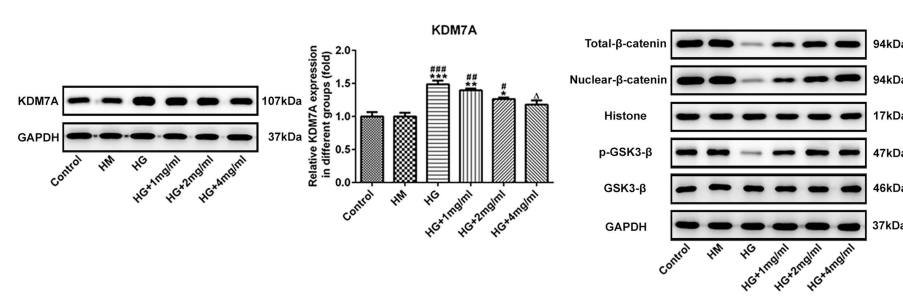

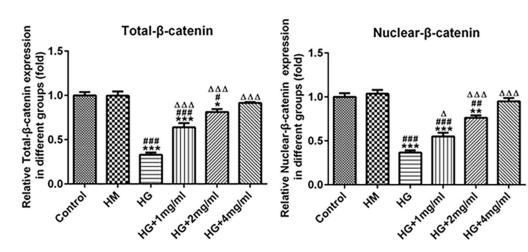
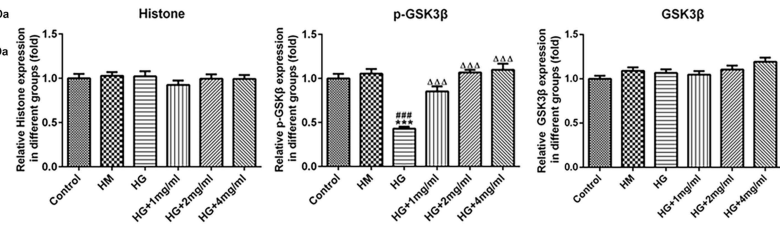

Figure 3 KDM7A expression is increased by HG induction, while KDM7A expression is inhibited by catalpol. (A) The Western blot analysis detected the KDM7A expression in HG-induced MC3T3-EI cells $(n=3)$. ${ }^{*} \mathrm{P}<0.05,{ }^{*} * \mathrm{P}<0.0 \mathrm{I}$ and ${ }^{*} * \mathrm{*} P<0.00 \mathrm{I}$ vs. control group. ${ }^{\#} \mathrm{P}<0.05$, ${ }^{\#} \mathrm{P}<0.0 \mathrm{I}$ and ${ }^{\# \#} \mathrm{P}<0.00 \mathrm{I}$ vs. $\mathrm{HM}$ group. ${ }^{\Delta} \mathrm{P}<0.05$ vs. $\mathrm{HG}$ group. (B) The expression of $\mathrm{Wnt} / \beta$-catenin signaling pathway in HG-induced MC3T3-EI cells was detected by Western blot analysis ( $\mathrm{n}=3$ ). $* \mathrm{P}<0.05$, $* * P<0.0 \mathrm{I}$ and ${ }^{* * *} \mathrm{P}<0.001$ vs. control group. ${ }^{\#} \mathrm{P}<0.05,{ }^{\# \#} \mathrm{P}<0.01$ and ${ }^{\# \#} \mathrm{P}<0.001$ vs. $\mathrm{HM}$ group. ${ }^{\Delta} \mathrm{P}<0.05$ and ${ }^{\Delta \Delta \Delta} \mathrm{P}<0.00$ I vs. HG group. 
catalpol promoted the expression of Total- $\beta$-catenin, Nuclear- $\beta$-catenin and p-GSK3 $\beta$ in a dose-dependent manner. The expression of GSK3 $\beta$ and histone in all groups was not changed (Figure 3B).

\section{Catalpol Regulates the Wnt/ $\beta$-Catenin Signaling Pathway Through KDM7A in MC3T3-EI Cells Induced by HG}

Cell transfection confirmed by Western blot analysis showed that the expression of KDM7A was down-regulated in MC3T3-E1 cells transfected with ShRNA-KDM7A-1 and ShRNA-KDM7A-2, and it was up-regulated in MC3T3-E1 cells transfected with
overexpression-KDM7A. The KDM7A expression in ShRNA-KDM7A-1-transfected MC3T3-E1 cells was lower than that in ShRNA-KDM7A-2-transfected MC3T3-E1 cells, so ShRNA-KDM7A-1 was used in the subsequent experiment (Figure 4A). HG decreased the expression of Total- $\beta$-catenin, Nuclear- $\beta$-catenin and $\mathrm{p}$-GSK3 $\beta$, and $4 \mathrm{mg} / \mathrm{kg}$ catalpol reversed the $\mathrm{HG}$ inhibition against the expression of Total- $\beta$-catenin, Nuclear- $\beta$-catenin and $p$-GSK3 $\beta$. The expression of Total- $\beta$-catenin, Nuclear- $\beta$-catenin and p-GSK3 $\beta$ in HGinduced MC3T3-E1 cells treated with $4 \mathrm{mg} / \mathrm{kg}$ catalpol was promoted by silencing of KDM7A and KDM7A overexpression (Figure 4B).
A

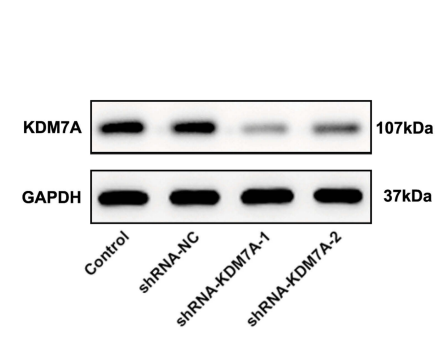

C

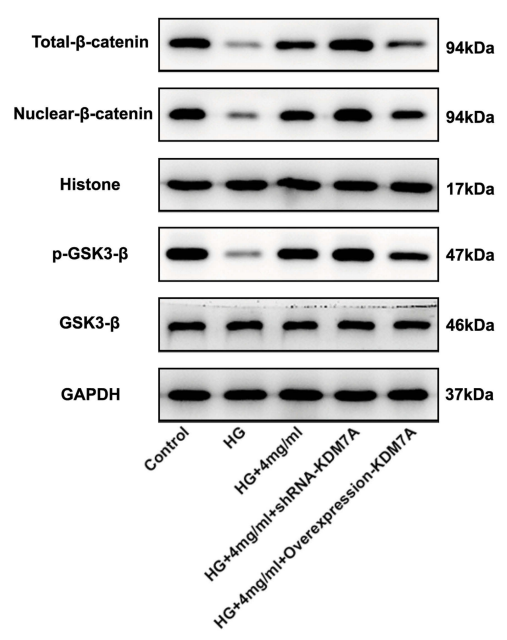

KDM7A
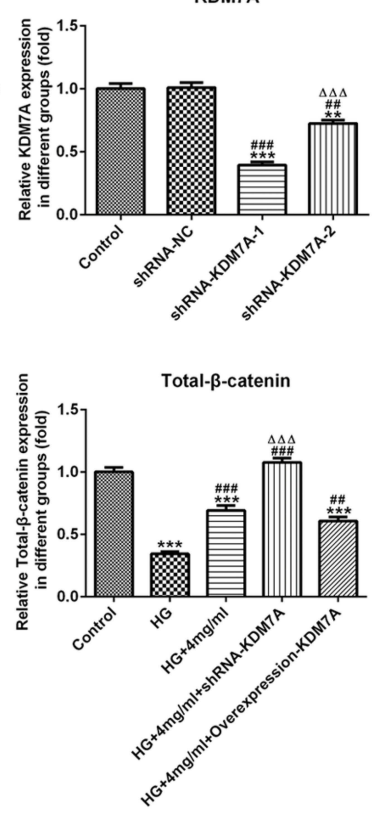

Histone

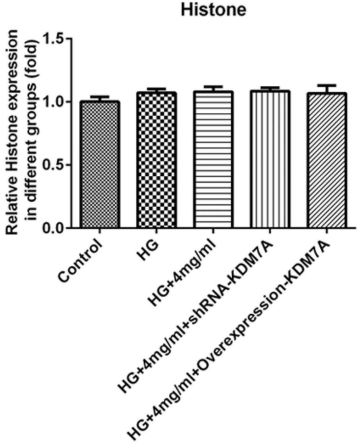

B
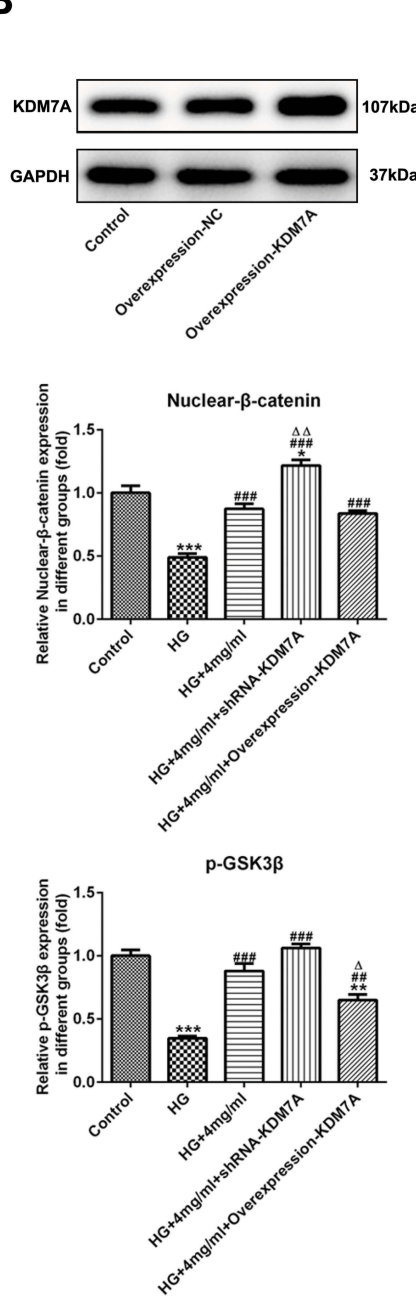

KDM7A
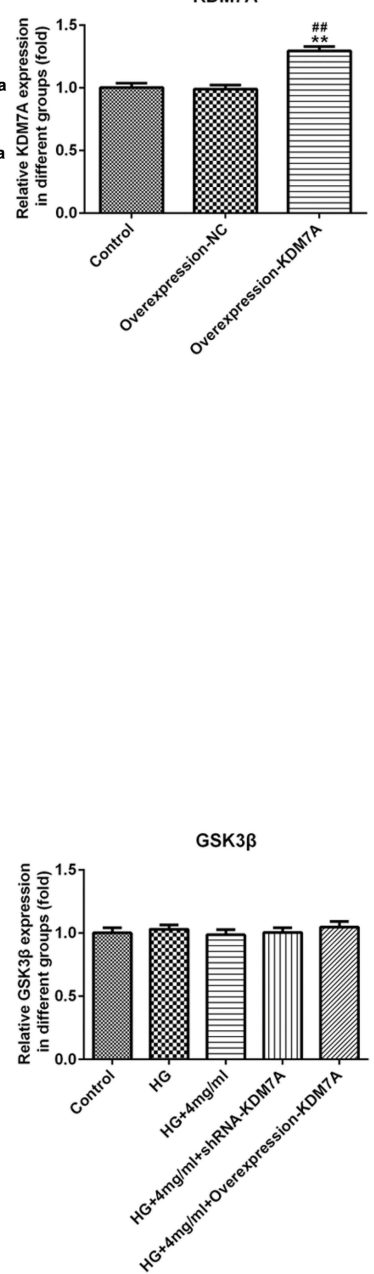

Figure 4 Catalpol regulates the Wnt/ $\beta$-catenin signaling pathway through KDM7A in MC3T3-EI cells induced by HG. (A, B) The transfection effects of KDM7A was verified by Western blot analysis $(n=3)$. ${ }^{* *} \mathrm{P}<0.01$ and ${ }^{* * *} \mathrm{P}<0.00 \mathrm{I}$ vs. control group. ${ }^{\#} \mathrm{P}<0.0 \mathrm{I}$ and ${ }^{\# \#} \mathrm{P}<0.00 \mathrm{I}$ vs. shRNA-NC group. ${ }^{\# \#} \mathrm{P}<0.0 \mathrm{I}$ vs. overexpression-NC group. ${ }^{\triangle \triangle A} \mathrm{P}<0.00 \mathrm{I}$ vs. shRNA-KDM7A-I group. (C) The expression of $W n t / \beta$-catenin signaling pathway in HG-induced MC3T3-EI cells after transfection was detected by Western blot analysis $(\mathrm{n}=3)$. ${ }^{*} \mathrm{P}<0.05$, ${ }^{* *} \mathrm{P}<0.0 \mathrm{I}$ and $* * * \mathrm{P}<0.00 \mathrm{I}$ vs. control group. ${ }^{\# \#} \mathrm{P}<0.0 \mathrm{I}$ and ${ }^{\# \#} \mathrm{P}<0.00 \mathrm{I}$ vs. $\mathrm{HG}$ group. ${ }^{\Delta} \mathrm{P}<0.05,{ }^{\Delta \Delta} \mathrm{P}<0.0 \mathrm{I}$ and ${ }^{\Delta \Delta \Delta} \mathrm{P}<0.00 \mathrm{I}$ vs. $\mathrm{HG}+4 \mathrm{mg} / \mathrm{mL}$ group. 
Catalpol Alleviates the Inhibition Effects of HG on Differentiation and Proliferation of MC3T3-EI Cells by Regulating the Wnt/ $\beta$ Catenin Signaling Pathway Through KDM7A

SKL2001 is used as a $\beta$-catenin agonist. HG induction inhibited cell proliferation, while $4 \mathrm{mg} / \mathrm{kg}$ catalpol improved the proliferation of HG-induced MC3T3-E1 cells. Silencing of KDM7A could further improve the proliferation ability of HG-induced MC3T3-E1 cells treated with $4 \mathrm{mg} / \mathrm{kg}$ catalpol. KDM7A overexpression inhibited the proliferation of HG-induced MC3T3-E1 cells treated with $4 \mathrm{mg} / \mathrm{kg}$ catalpol compared with $\mathrm{HG}+4 \mathrm{mg} /$ $\mathrm{mL}$ group while SKL2001 improved the proliferation of HG-induced MC3T3-E1 cells (Figure 5A). HG induction decreased the mineralized nodules and $4 \mathrm{mg} / \mathrm{kg}$ catalpol increased the mineralized nodules in HG-induced MC3T3-E1 cells. The mineralized nodules in HGinduced MC3T3-E1 cells treated with $4 \mathrm{mg} / \mathrm{kg}$ catalpol continued to increase when they were transfected with ShRNA-KDM7A while decreased when they were transfected with overexpression-KDM7A. And, SKL2001 further increased the mineralized nodules in HG-induced MC3T3-E1 cells (Figure 5B). The expression of RUNX2, Collagen I, OCN, BMP4 and BMP7 in MC3T3-E1 cells was decreased when induced by $\mathrm{HG}$ while increased when treated with $4 \mathrm{mg} / \mathrm{kg}$ catalpol. Silencing of KDM7A enhanced the expression of RUNX2, Collagen I, OCN, BMP4 and BMP7 in HG-induced MC3T3-E1 cells treated with $4 \mathrm{mg} / \mathrm{kg}$ catalpol. KDM7A overexpression suppressed the expression of these proteins while SKL2001 could reverse this effect (Figure 5C). HG significantly decreased the ALP activity in MC3T3-E1 cells, which was reversed by the treatment of $4 \mathrm{mg} / \mathrm{kg}$ catalpol. Silencing of KDM7A further improved the ALP activity of HG-induced MC3T3-E1 cells treated with $4 \mathrm{mg} / \mathrm{kg}$ catalpol. KDM7A overexpression inhibited the ALP activity while SKL2001 improved the ALP activity of HG-induced MC3T3-E1 cells (Figure 5D).

\section{Discussion}

Here, we explored whether catalpol enhanced the proliferation and differentiation of MC3T3 cells induced by high glucose through $\mathrm{Wnt} / \beta$-catenin signaling pathway through KDM7A. It was shown that catalpol promoted the proliferation and differentiation by activating the Wnt/ $\beta$-catenin signaling pathway through suppressing the KDM7A expression.
KDM7A, also known as JHDM1D, contains a JmjC domain which can remove the enzyme activity of histone methylation markers. ${ }^{13-16}$ KDM7A promotes the development of nerve and brain by removing dimethylated markers of $\mathrm{H} 3 \mathrm{~K} 9 \mathrm{me} 2$ and $\mathrm{H} 3 \mathrm{~K} 27 \mathrm{me} 2$ in the FGF4 promoter regions. ${ }^{17}$ In the hunger state, the up-regulated expression of KDM7A can inhibit the expression of angiogenic factors, thereby inhibiting the formation of blood vessels and tumor growth. ${ }^{18}$ Son et $\mathrm{al}^{19}$ found that KDM7A was involved in the differentiation of brown fat cells. Research has shown that mRNA expression of KDM7A is up-regulated during the adipogenic differentiation of ST2 cells. Overexpression of KDM7A wild type plasmid may promote the differentiation of ST2 cells into adipocytes and inhibit the differentiation of ST2 cells into osteoblasts. ${ }^{20}$ In this study, HG promoted the expression of KDM7A while catalpol inhibited the expression of KDM7A. And, the differentiation of MC3T3-E1 cells was inhibited in HG-induced MC3T3E1 cells, which could be reversed by catalpol treatment. Silencing of KDM7A in HG-induced MC3T3-E1 cells treated with catalpol could further enhance the differentiation of MC3T3-E1 cells, which was reversed by KDM7A overexpression. Hence, KDM7A inhibition plays a positive role in promoting KDM7A expression, which is consistent with the published article.

As an important transcription factor in osteoblastic differentiation, Runx2 is involved in osteoblastic and chondrogenic differentiation. Its expression is regulated by the Wnt and BMP signaling pathways. ${ }^{21}$ However, contrary to the role in regulating osteoblastic differentiation as an early transcription factor, Runx 2 may inhibit osteoblastic differentiation in late differentiation. ${ }^{22}$ Here, Runx2 expression was increased when the differentiation of MC3T3-E1 cells was increased. Activation of Wnt/ $\beta$ catenin signaling pathway can promote osteoblast differentiation and proliferation. ${ }^{23}$ In precursor osteoblasts, deficiency of $\beta$-catenin causes the development of osteoblast stagnating at an early stage, thereby leading to defective embryonic skeletal. ${ }^{24}$ In developing osteoblasts, lack of $\beta$-catenin leads to impaired differentiation and mineralization. ${ }^{25}$ The Wnt/ $\beta$-catenin signaling pathway can alter the ratio of OPG/RANKL in mature and precursor osteoblasts, thereby reducing bone absorption. ${ }^{26}$ Here, Wnt/ $\beta$-catenin expression was activated by SKL2001. KDM7A overexpression inhibited the differentiation of HG-induced MC3T3-E1 cells 
A

C

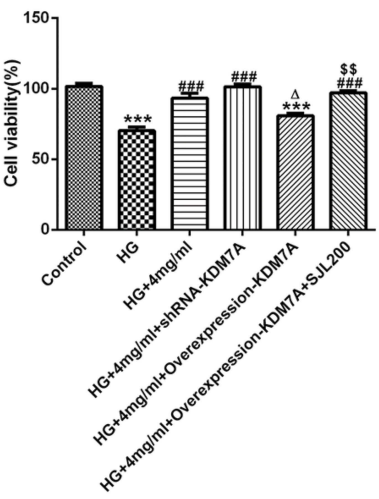

B
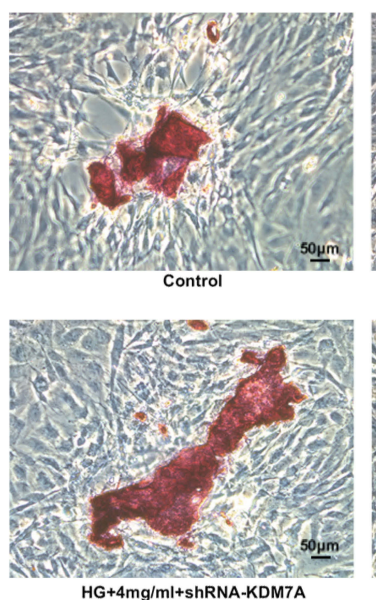

HG+4mg/ml+shRNA-KDM7A

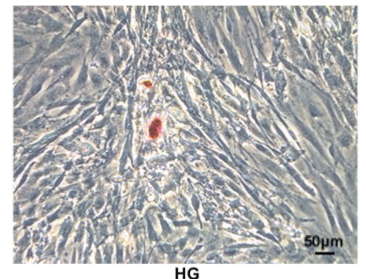

HG

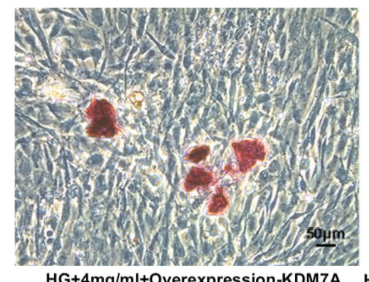

$\mathrm{HG}+4 \mathrm{mg} / \mathrm{ml}+$ Overexpression-KDM7A $\mathrm{HG}+4 \mathrm{mg} / \mathrm{ml}+$ Overexpression-KDM7A+SJL200
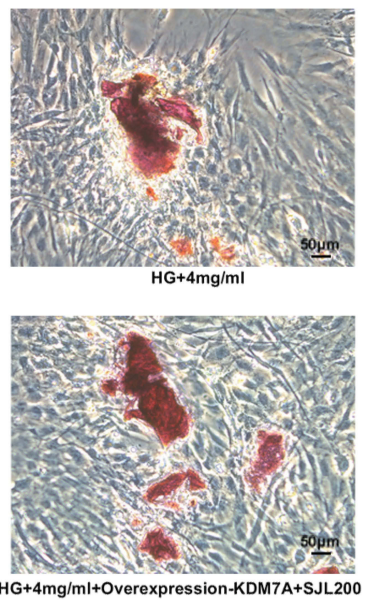

Collagen1

OCN

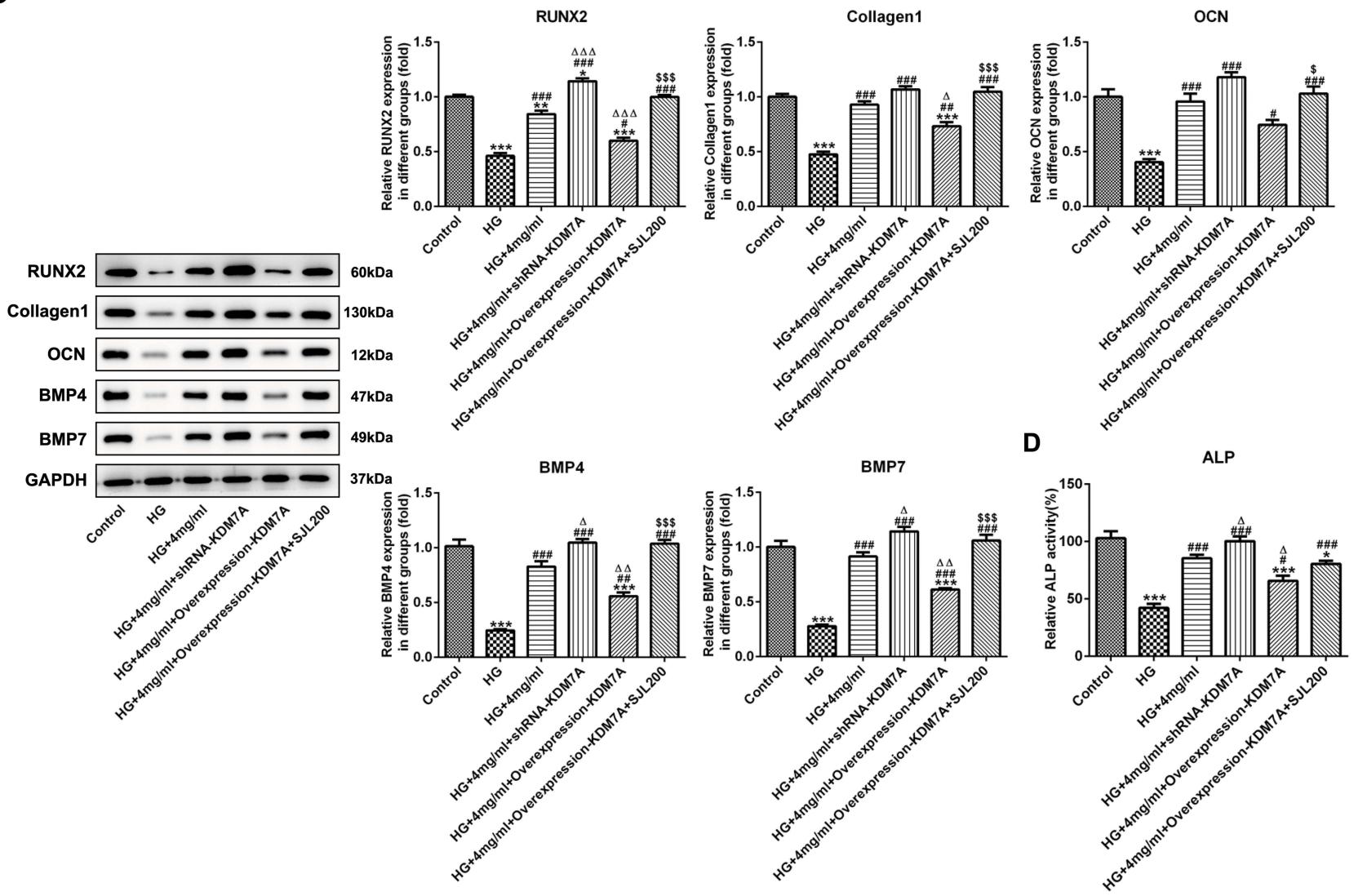

Figure 5 Catalpol alleviates the inhibition effects of HG on differentiation and proliferation of MC3T3-EI cells by regulating the Wnt/ $\beta$-catenin signaling pathway through KDM7A. (A) The proliferation of HG-induced MC3T3-EI cells after transfection was determined by CCK-8 assay. $* * * P<0.001$ vs. control group. $\mathrm{P}<0.00 \mathrm{I}$ vs. $H G$ group. ${ }^{\Delta} \mathrm{P}<0.05$ vs. $\mathrm{HG}+4 \mathrm{mg} / \mathrm{mL}$ group. ${ }^{\$ \$} \mathrm{P}<0.01$ vs. $\mathrm{HG}+4 \mathrm{mg} / \mathrm{mL}+$ overexpression-KDM7A group (magnification of 200 ). (B) The differentiation of $\mathrm{HG}$ induced MC3T3-EI cells after transfection was observed by alizarin red staining. (C) The expression of RUNX2, Collagen I, OCN, BMP4 and BMP7 in HG-induced MC3T3-EI cells after transfection was detected by Western blot analysis $(n=3)$. $* P<0.05, * * P<0.01$ and $* * * P<0.001$ vs. control group. ${ }^{\#} P<0.05$, \#\# $P<0.01$ and

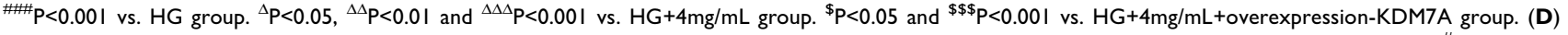
The ALP activity in HG-induced MC3T3-EI cells after transfection was assessed by the ALP assay kit. $* \mathrm{P}<0.05$ and $* * * P<0.001$ vs. control group. ${ }^{*} \mathrm{P}<0.05$ and ${ }^{\#} \mathrm{P}<0.00$ I vs. $\mathrm{HG}$ group. ${ }^{\Delta} \mathrm{P}<0.05$, vs. $\mathrm{HG}+4 \mathrm{mg} / \mathrm{mL}$ group.

treated with catalpol, which could be promoted by SKL2001.

In conclusion, catalpol improved the proliferation and differentiation of MC3T3 cells induced by HG by activating the $\mathrm{Wnt} / \beta$-catenin signaling pathway by inhibiting the expression of KDM7A. The new findings may provide theoretical instructions for DOP clinically. 


\section{Funding}

Natural Science Foundation of Jiangsu Province (BK20180996); Project of Jiangsu Bureau of traditional Chinese Medicine (YB201964) and the 16th batch of "Six Talent Peak" project of Jiangsu Province (WSN-282).

\section{Disclosure}

The authors declare they have no competing interests.

\section{References}

1. Zimmet PZ, Magliano DJ, Herman WH, et al. Diabetes: a 21st century challenge. Lancet Diabetes Endocrinol. 2014;2(1):56-64. doi:10.1016/S2213-8587(13)70112-8

2. Zimmet P, Alberti KG, Magliano DJ, et al. Diabetes mellitus statistics on prevalence and mortality: facts and fallacies. Nat Rev Endocrinol. 2016;12(10):616-622. doi:10.1038/nrendo.2016.105

3. Li XJ, Zhu Z, Han S-L, et al. Bergapten exerts inhibitory effects on diabetes-related osteoporosis via the regulation of the PI3K/AKT, JNK/MAPK and NF- $\mathrm{KB}$ signaling pathways in osteoprotegerin knockout mice. Int J Mol Med. 2016;38(6):1661-1672.

4. Chen Z, Zhao GH, Zhang YK, et al. Research on the correlation of diabetes mellitus complicated with osteoporosis with lipid metabolism, adipokines and inflammatory factors and its regression analysis. Eur Rev Med Pharmacol Sci. 2017;21(17):3900-3905.

5. Zhang RX, Li M-X, Jia Z-P. Rehmannia glutinosa: review of botany, chemistry and pharmacology. J Ethnopharmacol2008. 117(2):0-214.

6. Zou GL, Zhong WL, Wu F, et al. Catalpol attenuates cardiomyocyte apoptosis in diabetic cardiomyopathy via Neat1/miR-140-5p/HDAC4 axis. Biochimie. 2019;165:90-99. doi:10.1016/j.biochi.2019.05.005

7. Lin C-M, Wang B-W, Fang W-J, et al. Catalpol ameliorates neointimal hyperplasia in diabetic rats. Planta Med. 2019;85(5):406-411.

8. Lin C, Shyu KG, Wang BW. Catalpol ameliorates neointimal hyperplasia in streptozotocin induced diabetic. Eur J Heart Fail. 2019;21:161.

9. Liu JY, Zheng CZ, Hao XP, et al. Catalpol ameliorates diabetic atherosclerosis in diabetic rabbits. Am J Transl Res. 2016;8:4278-4288.

10. Zhu Y, Wang YM, Jia YC, et al. Catalpol promotes the osteogenic differentiation of bone marrow mesenchymal stem cells via the Wnt/-catenin pathway. Stem Cell Res Ther. 2019;10:14. doi:10. 1186/s13287-019-1143-y

11. Chen ZM, Lu M, Zhao FJ, et al. Catalpol promotes proliferation and osteogenic differentiation of rat bone marrow stromal cells. Int J Clin Exp Med. 2017;10(8):11929-11936.

12. Yang X, Wang G, Wang Y, et al. Histone demethylase KDM7A reciprocally regulates adipogenic and osteogenic differentiation via regulation of $\mathrm{C} / \mathrm{EBP} \alpha$ and canonical Wnt signalling. J Cell Mol Med. 2019. 23(3):2149-2162. doi: $10.1111 / \mathrm{jcmm} .14126$
13. Jenuwein T, Allis C. Translating the histone code. Science. 2001;293:1074-1080. doi:10.1126/science.1063127

14. Tsukada YI, Fang J, Erdjument-bromage H, et al. Histone demethylation by a family of JmjC domain-containing proteins. Nature. 2005;439(7078):811-816. doi:10.1038/nature04433

15. Pfau R, Tzatsos A, Kampranis S, et al. Members of a family of JmjC domain-containing oncoproteins immortalize embryonic fibroblasts via a JmjC domain-dependent process. Proc Natl Acad Sci U S A. 2008;105:1907-1912. doi:10.1073/pnas.0711865105

16. Yapeng H, Li X, Cheng L, et al. Genome-wide analysis of soybean JmjC domain-containing proteins suggests evolutionary conservation following whole-genome duplication. Front Plant Sci. 2016;7: 1800.

17. Huang C, Xiang Y, Wang Y, et al. Dual-specificity histone demethylase KIAA1718 (KDM7A) regulates neural differentiation through FGF4. Cell Res. 2010;20(2):154-165. doi:10.1038/cr.2010.5

18. Osawa T, Muramatsu M, Wang F, et al. Increased expression of histone demethylase JHDM1D under nutrient starvation suppresses tumor growth via down-regulating angiogenesis. Proc Natl Acad Sci U S A. 2011;108(51):20725-20729. doi:10.1073/pnas.1108462109

19. Son MJ, Kim WK, Oh KJ, et al. Methyltransferase and demethylase profiling studies during brown adipocyte differentiation. BMB Rep. 2016;49(7):388-393. doi:10.5483/BMBRep.2016.49.7.062

20. Yang X. The Role of Histone Demethylase KDM7A in Adipogenic and Osteogenic Commitment of Mesenchymal Stem Cells and the Mechanisms Involved. Tianjin Medical University; 2017.

21. Liu W, Toyosawa S, Furuichi T, et al. Overexpression of Cbfa1 in osteoblasts inhibits osteoblast maturation and causes osteopenia with multiple fractures. J Cell Biol. 2001;155:157-166. doi:10.1083/ jcb.200105052

22. Sinha K, Zhou X. Genetic and molecular control of osterix in skeletal formation. J Cell Biochem. 2013;114(5):975-984.

23. Canalis E. Management of endocrine disease: novel anabolic treatments for osteoporosis. Eur J Endocrinol. 2017;178:EJE-17. doi:10. 1530/EJE-17-0920

24. Glass DA II, Bialek P, Ahn JD, et al. Canonical Wnt signaling in differentiated osteoblasts controls osteoclast differentiation. Dev Cell. 2005;8(5):751-764. doi:10.1016/j.devcel.2005.02.017

25. Nie B, Zhou S, Fang X, Li W, Wang B, Guan S. Implication of receptor activator of NF- $\mathrm{KB}$ ligand in $\mathrm{Wnt} / \beta$-catenin pathway promoting osteoblast-like cell differentiation. J Huazhong Univ Sci Technolog Med Sci. 2012;32(6):818-822. doi:10.1007/s11596-0121040-4

26. Ross SE, Erickson RL, Gerin I, et al. Microarray analyses during adipogenesis: understanding the effects of Wnt signaling on adipogenesis and the roles of liver $\mathrm{X}$ receptor alpha in adipocyte metabolism. Mol Cell Biol. 2002;22(16):5989-5999. doi:10.1128/ MCB.22.16.5989-5999.2002

\section{Publish your work in this journal}

Diabetes, Metabolic Syndrome and Obesity: Targets and Therapy is an international, peer-reviewed open-access journal committed to the rapid publication of the latest laboratory and clinical findings in the fields of diabetes, metabolic syndrome and obesity research. Original research, review, case reports, hypothesis formation, expert opinion and commentaries are all considered for publication. The manuscript management system is completely online and includes a very quick and fair peer-review system, which is all easy to use. Visit http://www.dovepress.com/testimonials.php to read real quotes from published authors. 